$$
\begin{aligned}
& \text { Pontifícia Universidade } \text { Católica }_{\text {a }} \\
& \text { DO RIO DE JANEIRO }
\end{aligned}
$$

Cláudia Barucke Marcondes Paes Leme

\title{
Utilização da Tecnologia RFID aplicada no Espectro Óptico para Avaliação dos Recursos Disponíveis em Anéis Metropolitanos e seu Impacto Econômico
}

\section{Tese de Doutorado}

Tese apresentada ao Programa de Pós-graduação em Engenharia Elétrica da PUC-Rio como requisito parcial para obtenção do título de Doutor em Engenharia Elétrica.

Orientadores: Marbey Manhães Mosso Abelardo Podcameni (in memoriam) 


$$
\text { Pontifícia }_{\begin{array}{r}
\text { Universidade } \\
\text { Do Rio de Janeiro }
\end{array}}
$$

Cláudia Barucke Marcondes Paes Leme

\section{Utilização da Tecnologia RFID Aplicada no Espectro Óptico para Avaliação dos Recursos Disponíveis em Anéis Metropolitanos e seu Impacto Econômico}

Tese apresentada como requisito parcial para obtenção do grau de Doutor pelo Programa de Pós-Graduação em Engenharia Elétrica do Departamento de Engenharia Elétrica do Centro Técnico Científico da PUC-Rio. Aprovada pela Comissão Examinadora abaixo assinada.

Prof. Marbey Manhães Mosso Orientador

Centro de Estudos de Telecomunicações - PUC-Rio

Prof. Gláucio Lima Siqueira Centro de Estudos de Telecomunicações - PUC-Rio

Profa. Maria Cristina Ribeiro Carvalho Centro de Estudos de Telecomunicações - PUC-Rio

Prof. Antonio Dias de Macedo Filho Marinha do Brasil

Prof. Luiz Alberto de Andrade Instituto Aeronáutica e Espaço/CTA

Prof. José Eugenio Leal Coordenador Setorial do Centro

Técnico Científico - PUC-Rio

Rio de Janeiro, 26 de março de 2009. 
Todos os direitos reservados. É proibida a reprodução total ou parcial do trabalho sem autorização da universidade, do autor e do orientador.

\section{Cláudia Barucke Marcondes Paes Leme}

Graduou-se em Engenharia Elétrica - ênfase em Telecomunicações pela PUC-Rio (Pontifícia Universidade Católica do Rio de Janeiro) em 1997. Mestre em Engenharia Elétrica pelo CETUC (Centro de Estudos em Telecomunicações da PUC-Rio) em 2000. Trabalhou 5 anos como engenheira de transmissão em uma operadora telefônica de longa distância e em uma multinacional de equipamentos e sistemas de telecomunicações, lidando com sistemas de gerência de redes de telecomunicações. Possui experiência na área de Engenharia Elétrica, ênfase em Sistemas de Telecomunicações Ópticos e de Microondas, atuando principalmente em: redes ópticas, sistemas de gerência de redes, sistemas de transporte de dados, RFID (identificação por rádio freqüência) e endereçamento de nós utilizando subportadoras elétricas de microondas.

Ficha Catalográfica

Leme, Cláudia Barucke Marcondes Paes

Utilização da tecnologia RFID aplicada no espectro óptico para avaliação dos recursos disponíveis em anéis metropolitanos e seu impacto econômico / Cláudia Barucke Marcondes Paes Leme ; orientadores: Marbey Manhães Mosso, Abelardo Podcameni (in memoriam). - 2009.

$111 \mathrm{f}$ : : il. ; $30 \mathrm{~cm}$

Tese (Doutorado em Engenharia Elétrica)-Pontifícia Universidade Católica do Rio de Janeiro, Rio de Janeiro, 2009.

Inclui bibliografia

1. Engenharia elétrica - Teses. 2. RFID. 2. Subportadoras de RF. 3. Anel óptico metropolitano. 4. Inteligência na camada física. 5. Gerência de rede. 6. Gerência de inventário. 7. NGN. 8. Cadeia de valores. 9. Análise de sensibilidade. I. Mosso , Marbey Manhães. II. Podcameni, Abelardo. III. Pontifícia Universidade Católica do Rio de Janeiro. Departamento de Engenharia Elétrica. IVI. Título.

CDD: 621.3 
Para minha família: meus pais Norma e Carlos, meus irmãos, meu marido Marcos e meus filhos Luiza e Mateus. E para o meu eterno orientador, o saudoso Prof. Abelardo Podcameni. 


\section{Agradecimentos}

Aos meus orientadores, Prof. Abelardo Podcameni (in memoriam) e Prof. Marbey Manhães Mosso, pela orientação na execução deste trabalho, pela dedicação, compreensão, apoio e pelos ensinamentos que me transmitiram.

Ao CNPq e à PUC-Rio, pelos auxílios concedidos, sem os quais este trabalho não poderia ter sido realizado.

À Prof. Maria Cristina Ribeiro Carvalho, pelo apoio e incentivo dado.

Aos colegas do Laboratório GSOM, em especial Rodolfo Araújo de Azevedo Lima, Vanessa Magri e Claumir Sarzeda, pela amizade e companheirismo.

Aos amigos da Alcatel-Lucent e da Intelig Telecomunicações, pelo apoio e pelas informações de pesquisa de campo.

Aos amigos, familiares e todas as pessoas que de uma forma ou de outra me apoiaram, estimularam ou me ajudaram.

A Deus, por me dar saúde e forças para vencer mais uma etapa em minha vida.

Aos meus pais, pela educação, incentivo, atenção e carinho de todas as horas.

E principalmente a meu marido e meus filhos, por dividirem meu tempo entre eles e a tese. E por me darem carinho e força para que eu finalizasse esse trabalho. 


\section{Resumo}

Leme, Cláudia Barucke Marcondes Paes. Utilização da Tecnologia RFID Aplicada no Espectro Óptico para Avaliação dos Recursos Disponíveis em Anéis Metropolitanos e seu Impacto Econômico. Rio de Janeiro, 2009. 111p. Tese de Doutorado - Departamento de Engenharia Elétrica, Pontifícia Universidade Católica do Rio de Janeiro.

Ao longo do presente trabalho é apresentado o desenvolvimento de um sistema operando na camada óptica física, que avalia o inventário de capacidade de um anel óptico metropolitano. O sistema de RFID tradicional, utilizado comercialmente para avaliação de estoques de uma cadeia de suprimentos, foi adaptado para descrever a capacidade disponível dos elementos de rede dos nós deste anel metropolitano. Para esta adaptação foi criado um código utilizando subportadoras de RF, denominado EPC Telecom, compatível com os procedimentos usuais aplicados na leitura e avaliação de etiquetas RFID tradicionais. A partir do sistema RFID assim desenvolvido, o conceito de cadeia de valores segundo Michael Porter é utilizado para avaliar o desempenho econômico de operadoras de telecomunicações e os possíveis impactos quando da utilização do sistema proposto.

\section{Palavras Chave}

RFID, subportadoras de RF, anel óptico metropolitano, inteligência na camada física, gerência de rede, gerência de inventário, NGN, cadeia de valores, análise de sensibilidade. 


\section{Abstract}

Leme, Cláudia Barucke Marcondes Paes. RFID Applied to Optical Spectrum for Metropolitan Ring Network Resources Inventory and its Economic Impact. Rio de Janeiro, 2009. 111p. D.Sc. Thesis Departamento de Engenharia Elétrica, Pontifícia Universidade Católica do Rio de Janeiro.

In this work, by using RFID, a technology globally employed in logistic and supply chain control, a system operating at optical physical layer implements a real time, reliable and distributed capacity inventory evaluation of metropolitan ring networks. This system is achieved by introducing a binary combination of RF subcarriers in the optical spectrum. The array of subcarriers is generated through a new proposed code, denominated EPC Telecom, compatible with traditional RFID codes. Also, the concept of value chain according Michael Porter is employed to evaluate the economic performance of telecommunications operators and the impact of this new RFID system in these operators.

\section{Keywords}

RFID, RF subcarriers, metropolitan optical rings, intelligent physical layer, network management, inventory management, NGN, value chain, sensitivity analysis. 


\section{Sumário}

1 Introdução 15

1.1. Cenário Atual do Gerenciamento de Redes de Telecomunicações 15

$\begin{array}{ll}\text { 1.2. Tecnologia RFID } & 18\end{array}$

2 Tecnologia de Identificação por Rádio Freqüência (RFID) 21

2.1. Como funciona? 21

2.1.1. Processo de leitura e interpretação de dados RFID 23

2.2. Freqüências de Operação 26

2.3. Tipos de Etiquetas $\quad 27$

2.3.1. Etiquetas Passivas $\quad 27$

2.3.2. Etiquetas Semi-Ativas (ou Semi-Passivas) 28

2.3.3. Etiquetas Ativas 29

2.4. Padrão do Código EPC 29

2.5. Principais Aplicações da Tecnologia RFID 31

2.5.1. Rastreamento de itens 31

2.5.2. Controle de inventário 32

2.5.3. Gerência de recursos 32

2.5.4. Sistemas antifurto 33

2.5.5. Pagamento eletrônico 33

2.5.6. Controle de acesso 34

2.5.7. Monitoração e sensoriamento para ambientes e pessoas 34

2.5.8. Sistemas antifalsificação 34

2.5.9. Sistemas de logística na cadeia de suprimentos 35

2.5.10. Exemplo de implementação do RFID na cadeia de suprimentos 36

2.6. Comentários e Conclusões do Capítulo 39

3 Gerência de Inventário utilizando RFID em Redes Ópticas 40

3.1. Descrição do Sistema Proposto 40

3.2. Introdução das Subportadoras de RF na Rede Óptica 42 
3.3. Geração do Código EPC Telecom 48

3.4. Exemplo de Aplicação 54

3.5. Simulação do Código Proposto em Software Tradicional de RFID 56

3.6. Comentários e Conclusões do Capítulo 72

4 Avaliação Econômica de Redes Legada e NGN 74

4.1. Conceito de Cadeia de Valores 74

4.1.1. Atividades de suporte $\quad 75$

4.1.2. Atividades primárias 75

4.2. Elaboração da Cadeia de Valores de Redes de Telecomunicações 76

4.3. Comentários e Conclusões do Capítulo 85

5 Impactos Econômicos do Sistema Proposto 87

5.1. Aspectos Gerais dos Sistemas de Gerenciamento 87

5.2. Exemplo: Tecnologia RFID Proposta como um Subsistema na Camada Física Óptica das Redes das Operadoras Avaliadas 89

5.3. Análise de Sensibilidade 90

5.3.1. Redução dos custos de operações de gerência 90

5.4. Gerenciamento Independente Usando a Tecnologia RFID Proposta 92

5.5. Comentários e Conclusões do Capítulo

6 Comentários Finais e Conclusões 95

6.1. Observações Finais 95

6.2. Principais Resultados Obtidos 96

6.3. Principais Vantagens do Sistema Desenvolvido 97

6.4. Principais Dificuldades para Implementar o Sistema Proposto 97

$\begin{array}{ll}\text { 6.5. Desenvolvimentos Futuros } & 98\end{array}$

$\begin{array}{ll}7 \text { Referências } & 99\end{array}$

8 Glossário 106 


\section{Lista de figuras}

Figura 1 - Conceito de gerência de rede com controle autonômico [6]. 17

Figura 2- Arquitetura de um sistema RFID. 21

Figura 3- Exemplos de Etiquetas (Tags). 22

Figura 4 - Diagrama de blocos de um leitor RFID. 23

Figura 5 - Esquema básico da arquitetura da rede EPCglobal. 25

Figura 6 - Freqüências utilizadas em sistemas RFID [20]. 26

Figura 7 - Diagrama para etiqueta passiva de RFID [20]. 28

Figura 8 - Diagrama para etiqueta semi-ativa de RFID [20]. 28

Figura 9 - Diagrama para etiqueta ativa de RFID [20]. 29

Figura 10 - Estrutura típica de dados de um código EPC 96 bits. 30

Figura 11 - Exemplo de implementação de RFID em Cadeia de Suprimentos, com indicação passo-a-passo.

Figura 12 - Portadora de RF principal para os nós k-1, k e k+1. 40

Figura 13 - Captação dos dados do nó local e geração do código EPC TELECOM.

Figura 14 - Código binário de endereçamento de subportadora.

Figura 15 - Conjunto de subportadoras convertido pela portadora principal do nó.

Figura 16 - Diagrama de blocos representando um nó genérico de uma rede óptica, utilizando subportadoras de RF inseridas no domínio óptico Processo de Transmissão.

Figura 17 - Espectro de freqüência da transmissão do EPC Telecom pelo anel óptico.

Figura 18 - Diagrama de blocos representando um nó genérico de uma rede óptica, utilizando subportadoras de RF inseridas no domínio óptico Processo de Recepção.

Figura 19 - Diagrama de blocos representando um nó genérico de uma rede óptica, utilizando subportadoras de RF inseridas no domínio óptico Processo Total.

Figura 20 - Conversor oficial da GS1 alemã para geração de códigos mundiais EPC, para os sistemas RFID tradicionais. 
Figura 21 - Estrutura de formação do código EPC Telecom.

Figura 22 - Exemplo de varredura do pacote de totalização transmitindo as informações dos NEs existentes no nó 1 do anel.

Figura 23 - Exemplo do sistema proposto.

Figura 24 - Diagrama do anel óptico usado na simulação.

Figura 25 - Diagrama da rede óptica demonstrando os elementos e suas capacidades de cada nó.

Figura 26 - Analogia entre encaminhamento do produto na cadeia de suprimentos e transporte do EPC Telecom.

Figura 27 - Tela do Portable Demo para inserção de dados SDH do nó 1.

Figura 28 - Tela do Portable Demo para inserção dos elementos de DADOS do nó 1.

Figura 29 - Tela do Portable Demo para inserção de dados SDH do nó 2.

Figura 30 - Tela do Portable Demo para inserção dos elementos de DADOS do nó 2.

Figura 31 - Tela do Portable Demo para inserção de dados SDH do nó 3.

Figura 32 - Tela do Portable Demo para inserção dos elementos de DADOS do nó 3.

Figura 33 - Tela do Portable Demo para inserção de dados SDH do nó 4.

Figura 34 - Tela do Portable Demo para inserção dos elementos de DADOS do nó 4.

Figura 35 - Tela do Portable Demo de visualização dos elementos cadastrados no nó 1.

Figura 36 - Tela do Portable Demo de visualização dos elementos cadastrados no nó 2.

Figura 37 - Tela do Portable Demo de visualização dos elementos cadastrados no nó 3.

Figura 38 - Tela do Portable Demo de visualização dos elementos cadastrados no nó 4.

Figura 39 - "Entrega" do elemento solicitado do nó 1 para o nó $2-1^{0}$ PASSO: Escolha do NE.

Figura 40 - "Entrega" do elemento solicitado do nó 1 para o nó $2-2^{\circ}$ PASSO: Leitor identifica ordem de entrega solicitada, na saída do nó 1.

Figura 41 - "Entrega" do elemento solicitado do nó 1 para o nó 2 - $3^{\circ}$ PASSO: "Transporte" dos dados do NE solicitado para o nó 2. 
Figura 42 - "Entrega" do elemento solicitado do nó 1 para o nó $2-4^{\circ}$ PASSO: "Entrega" na entrada do nó 2, a ordem solicitada.

Figura 43 - "Entrega" do elemento solicitado do nó 1 para o nó 2 - 50 PASSO: Leitor identifica recebimento da informação, na entrada do nó 2.

Figura 44 - "Entrega" do elemento solicitado do nó 1 para o nó 2 - $6^{\circ}$ PASSO: Dados enviados pelo nó 1 , foram recebidos e armazenados na base de dados do nó 2 .

Figura 45 - Conceito de Cadeia de Valores segundo Porter [62].

Figura 46 - Distribuição das atividades das operadoras de acordo com a Cadeia de Valores segundo Porter.

Figura 47 - Avaliação econômica da operadora Bell South Florida em 1996, valores anuais em US dólares normalizados por linhas de acesso ativadas.

Figura 48 - Avaliação econômica da operadora Bell South Florida em 2007, valores anuais em US dólares normalizados por linhas de acesso ativadas.

Figura 49 - Avaliação econômica da operadora Bell South Georgia em 1996, valores anuais em US dólares normalizados por linhas de acesso ativadas.

Figura 50 - Avaliação econômica da operadora Bell South Georgia em 2007, valores anuais em US dólares normalizados por linhas de acesso ativadas.

Figura 51 - Avaliação econômica da operadora Pacific Bell California em 1996, valores anuais em US dólares normalizados por linhas de acesso ativadas.

Figura 52 - Avaliação econômica da operadora Pacific Bell California em 2007, valores anuais em US dólares normalizados por linhas de acesso ativadas.

Figura 53 - Distribuição média dos custos segundo a cadeia de valores em 1996.

Figura 54 - Distribuição média dos custos segundo a cadeia de valores em 2007.

Figura 55 - Separação dos custos de gerência e outros custos das operadoras em 1996.

Figura 56 - Separação dos custos de gerência e outros custos das operadoras em 2007.

Figura 57 - Introdução do sistema RFID aplicado no meio óptico físico.

Figura 58 - Análise de sensibilidade sobre os custos de Gerência em 2007, considerando a redução de custos proposta no exemplo do item 5.2. 
Figura 59 - Projeção da Redução dos Custos de Gerência considerando as três operadoras.

Figura 60 - Arquitetura do sistema EPC Telecom proposto. 


\section{Lista de tabelas}

Tabela 1 - Classificação em faixas percentuais da capacidade ocupada dos NEs.

Tabela 2. Códigos binários gerados para cada associação de protocolo, taxa e capacidade de utilização.

Tabela 3. Códigos binários gerados para indicar 32 diferentes nós de uma rede óptica.

Tabela 4. Descrição dos Elementos de Rede e respectivos códigos EPC Telecom de acordo com a capacidade utilizada no anel 1 SDH.

Tabela 5. Descrição dos Elementos de Rede e respectivos códigos EPC Telecom de acordo com a capacidade utilizada no anel 2 DADOS. 